\title{
Candidate genes for idiopathic epilepsy in four dog breeds
}

\author{
Kari J Ekenstedt ${ }^{*}$, Edward E Patterson ${ }^{1}$, Katie M Minor ${ }^{2}$ and James R Mickelson ${ }^{2}$
}

\begin{abstract}
Background: Idiopathic epilepsy (IE) is a naturally occurring and significant seizure disorder affecting all dog breeds. Because dog breeds are genetically isolated populations, it is possible that IE is attributable to common founders and is genetically homogenous within breeds. In humans, a number of mutations, the majority of which are genes encoding ion channels, neurotransmitters, or their regulatory subunits, have been discovered to cause rare, specific types of IE. It was hypothesized that there are simple genetic bases for IE in some purebred dog breeds, specifically in Vizslas, English Springer Spaniels (ESS), Greater Swiss Mountain Dogs (GSMD), and Beagles, and that the gene(s) responsible may, in some cases, be the same as those already discovered in humans.

Results: Candidate genes known to be involved in human epilepsy, along with selected additional genes in the same gene families that are involved in murine epilepsy or are expressed in neural tissue, were examined in populations of affected and unaffected dogs. Microsatellite markers in close proximity to each candidate gene were genotyped and subjected to two-point linkage in Vizslas, and association analysis in ESS, GSMD and Beagles.

Conclusions: Most of these candidate genes were not significantly associated with IE in these four dog breeds, while a few genes remained inconclusive. Other genes not included in this study may still be causing monogenic IE in these breeds or, like many cases of human IE, the disease in dogs may be likewise polygenic.
\end{abstract}

\section{Background}

The prevalence of epilepsy in humans is reported to be in the range of $4-10 / 1000$ in most study settings [1], with idiopathic epilepsy (IE) representing $15-20 \%$ of these cases [2]. It is now generally accepted that IE in humans is due to an underlying genetic origin [2], although causative mutations have been discovered in only a small subset of IEs, mostly in isolated populations. These identified epilepsy mutations are, for the most part, Mendelian or monogenic IEs [3-6], and are often termed "channelopathies" due to their occurrence in ion channel genes. In a recent review, 16 of 21 susceptibility genes for human epilepsy were ion channels or neurotransmitter receptors [7].

Canine epilepsy is a naturally occurring, spontaneous condition. Canine seizures exhibit a remarkable resemblance to human seizures [8] and the usefulness of naturally occurring canine epilepsy as a translational model to

\footnotetext{
* Correspondence: eken0003@umn.edu

'Department of Veterinary Clinical Sciences, College of Veterinary Medicine, University of Minnesota, 1352 Boyd Avenue, Saint Paul, Minnesota, 55108, USA

Full list of author information is available at the end of the article
}

explore potential treatments for human epilepsy was recently proposed by Leppik, et al. [9]. The prevalence of canine epilepsy is estimated to be between $0.5 \%$ and $5.7 \%$ and it is the most common chronic neurological disorder in dogs [10]. A diagnosis of IE in the canine indicates recurrent seizures for which no cause can be identified and implies a genetic predisposition [11]. While the molecular basis of IE in canines is entirely unknown, a hereditary basis for IE has been suggested in a number of dog breeds including Beagles [12], British Alsatians [13], Keeshonds [14], Labrador retrievers [15], Golden retrievers $[16,17]$, Bernese mountain dogs [18], Belgian Tervurens $[19,20]$, Boxers [21], Shetland sheepdogs [22], Vizslas [23], English Springer Spaniels [24], Irish wolfhounds [25], and Standard Poodles [26]. One inherited progressive seizure disorder that has been identified in dogs is the autosomal recessive Lafora disease in Miniature Wirehaired Dachshunds [27]. This progressive myoclonic epilepsy disorder results from a mutation in the $E P M 2 B$ (also known as NHLRC1-NHL repeat containing 1) gene.

Modes of inheritance predicted from examination of pedigrees of dogs with IE show evidence for a gene of
C Biomed Central

() 2011 Ekenstedt et al; licensee BioMed Central Ltd. This is an Open Access article distributed under the terms of the Creative Commons Attribution License (http://creativecommons.org/licenses/by/2.0), which permits unrestricted use, distribution, and reproduction in any medium, provided the original work is properly cited. 
major effect or autosomal recessive inheritance, both of which could occur due to a founder effect. Examples include the English Springer Spaniel (partially penetrant autosomal recessive or polygenic inheritance) [24], the Vizsla (autosomal recessive or polygenic inheritance) [23], and the Golden Retriever [16] and the Bernese Mountain Dog [18] (both autosomal recessive but controlled by more than one gene). There is enough variation in mode of inheritance and clinical characteristics in dogs to presume that the underlying genetic basis is unlikely to be identical between breeds.

Linebreeding and inbreeding have become commonplace to meet the very specific dog breed standards of the hundreds of dog breeds in existence today. These practices, while creating more uniform offspring, can also result in deleterious diseases due to the concentration of recessive mutations passed from the founders. Almost half of the nearly four hundred genetic disorders that have been described in dogs are recognized exclusively in one or a few breeds [28], presumably due to a strong founder effect.

The aim of the present study was to take advantage of the likely founder effect occurring in purebred dogs with IE in order to investigate an underlying genetic basis for IE in four dog breeds: the Vizsla, the English Springer Spaniel (ESS), the Greater Swiss Mountain Dog (GSMD) and the Beagle. A candidate gene approach was utilized; genes known to be involved in inherited human epilepsy, along with two genes involved in mouse epilepsy models and additional related gene family members, were selected. Most candidate genes tested were ion channels. Microsatellite markers within or very close to these genes were used to generate genotypes which were then subjected to either two-point linkage analysis or association chi-square studies. Using four different breeds, which may each have different forms of IE, increased the chances of potentially finding an associated gene in at least one of the breeds.

\section{Results}

Call rates in each sample set for the microsatellite markers that were ultimately used in each analysis were approximately $90 \%$ or higher. Heterozygosity was recorded for each marker as a measure of that marker's extent of utility for this study. Average heterozygosity \pm standard error of all markers analyzed for Beagles was $0.574 \pm 0.024$, for Vizslas was $0.569 \pm 0.026$, for ESS was $0.425 \pm 0.030$, and for GSMD was $0.341 \pm 0.030$. As expected, the average heterozygosity varied by breed and markers that were informative in one breed were not necessarily so in another breed. Out of all tested microsatellites, there were greater numbers of low heterozygosity (cut-off of 0.3 ) markers in the GSMD (27) and ESS (20) compared to the Vizslas (9) and Beagles (6), resulting in the lower average heterozygosities for the GSMD and ESS. For some genes, despite testing several microsatellites, results remain uninformative. For example, GABRD remains inconclusive in the ESS, despite testing three different microsatellites.

From the list of 52 candidate genes, 20 are known to be associated with inherited human epilepsy (indicated by a bolded entry with " in the tables) and two are associated with inherited mouse epilepsy models (indicated by a bolded entry with \# in the tables). All 20 of the inherited human epilepsy-associated genes and both mouse model genes were analyzed at least once in all four of the test breeds, except for CHRNA2 in the Vizslas. However, not all of the other 30 genes were examined in every breed.

In the two-point linkage study, the two large Vizsla families were divided into ten smaller families to decrease complexity and inbreeding loops. The power of this ten family pedigree to detect significant linkage was demonstrated with simulated linkage analysis. The highest average LOD score for simulated linkage was 3.63, obtained by using a simulated marker with four alleles, no heterogeneity, and a recombination frequency of 0.001 . The maximum attainable LOD score in these simulations was 8.46 , obtained by using a simulated marker with three alleles, $5 \%$ heterogeneity, and a recombination frequency of 0.001 . All of the simulation combinations obtained average LOD scores above 2.0 (suggestive linkage), demonstrating that the pedigrees are sufficient to detect linkage for a monogenic mutation. Results for the Vizslas are shown in Table 1 . The majority of the candidate gene loci have very negative LOD scores and 43 genes were successfully excluded from being linked with IE in this breed. All of the human epilepsy-associated genes, with the exception of CHRNA2, and both of the mouse model genes were excluded. Four genes (CACNA1I, CACNB3, CHRNA1, and SCN11A) had low heterozygosity or monomorphism for the markers tested, therefore, these genes cannot be excluded. In addition, two genes (CACNB1 and CACNB2) had LOD values falling between -2.0 and 3.2 , a grey zone of being neither linked nor unlinked; thus, conclusions cannot be drawn concerning possible linkage of these genes to IE in Vizslas. The most interesting of these was CACNB1, which had a LOD of 0.820 and a heterozygosity of 0.548 .

Results for the English Springer Spaniels are shown in Table 2. Pearson chi-square p-values are reported for this nonparametric association study. The majority of the loci $(\mathrm{n}=36)$ had highly insignificant $\mathrm{p}$-values, decreasing the likelihood that these genes are associated with IE in this breed; eighteen of the inherited human epilepsy genes were in this group, as were both of the mouse epilepsy genes. Nine genes, two of which are human epilepsyassociated genes (GABRD and CHRNA2), had markers with heterozygosities falling below the 0.3 cut-off, prohibiting the drawing of conclusions about these genes. One 
Table 1 Vizsla two-point linkage analysis results

\begin{tabular}{|c|c|c|c|c|}
\hline Gene Name & Designator & $\mathrm{H}$ & $\begin{array}{c}\text { LOD at } 0 \mathrm{cM} \\
(10 \mathrm{Fam})\end{array}$ & Results \\
\hline${ }^{*}$ ARX & First Set & 0.555 & $(-) 16.99$ & Excluded \\
\hline${ }^{*}$ CACNA1A & First Set & 0.163 & $(-)$ & Low Heterozygosity \\
\hline${ }^{*}$ CACNA1A & Second Set & 0.180 & $(-)$ & Low Heterozygosity \\
\hline${ }^{*}$ CACNA1A & Third Set & 0.142 & $(-)$ & Low Heterozygosity \\
\hline${ }^{*}$ CACNA1A & Fourth Set & 0.659 & $(-) 8.06$ & Excluded \\
\hline CACNA1B & First Set & 0.757 & $(-) 7.78$ & Excluded \\
\hline CACNA1D & First Set & 0.725 & $(-) 16.18$ & Excluded \\
\hline CACNA1E & First Set & 0.783 & $(-) 11.03$ & Excluded \\
\hline CACNA1F & First Set & 0.655 & $(-) 23.95$ & Excluded \\
\hline CACNA1G & First Set & 0.584 & $(-) 12.33$ & Excluded \\
\hline${ }^{*} \mathrm{CACNA} 1 \mathrm{H}$ & First Set & 0.000 & $(-)$ & Monomorphic \\
\hline${ }^{*} \mathrm{CACNA} 1 \mathrm{H}$ & Second Set & 0.423 & $(-) 7.59$ & Excluded \\
\hline CACNA1I & First Set & 0.275 & $(-)$ & Low Heterozygosity \\
\hline \#CACNA2D2 & First Set & 0.495 & $(-) 1.34$ & Neither Linked Nor Unlinked \\
\hline \#CACNA2D2 & Second Set & 0.665 & $(-) 6.88$ & Excluded \\
\hline CACNB1 & First Set & 0.548 & 0.82 & Neither Linked Nor Unlinked \\
\hline CACNB2 & First Set & 0.670 & $(-) 1.39$ & Neither Linked Nor Unlinked \\
\hline CACNB3 & First Set & 0.203 & $(-)$ & Low Heterozygosity \\
\hline *CACNB4 & First Set & 0.781 & $(-) 6.08$ & Excluded \\
\hline \#CACNG2 & First Set & 0.739 & $(-) 21.61$ & Excluded \\
\hline CACNG3 & First Set & 0.628 & $(-) 10.69$ & Excluded \\
\hline CACNG4 & First Set & 0.615 & $(-) 8.70$ & Excluded \\
\hline CACNG6 & First Set & 0.530 & $(-) 8.35$ & Excluded \\
\hline CHRNA1 & First Set & 0.291 & $(一)$ & Low Heterozygosity \\
\hline CHRNA3 & First Set & 0.629 & $(-) 3.58$ & Excluded \\
\hline${ }^{*}$ CHRNA4 and KCNQ2 & See KCNQ2 & & & \\
\hline CHRNA7 & First Set & 0.695 & $(-) 9.65$ & Excluded \\
\hline CHRNA9 & First Set & 0.420 & $(-) 4.54$ & Excluded \\
\hline CHRNA10 & First Set & 0.795 & $(-) 6.43$ & Excluded \\
\hline CHRNB1 & First Set & 0.587 & $(-) 4.62$ & Excluded \\
\hline${ }^{*}$ CHRNB2 & First Set & 0.725 & $(-) 18.39$ & Excluded \\
\hline CHRND & First Set & 0.655 & $(-) 5.05$ & Excluded \\
\hline${ }^{*} \mathrm{CLCN} 2$ & First Set & 0.665 & $(-) 4.75$ & Excluded \\
\hline DNM1 & First Set & 0.495 & $(-) 3.63$ & Excluded \\
\hline DNM1 & Fourth Set & 0.355 & $(-) 3.77$ & Excluded \\
\hline DNM1 & Fifth Set & 0.677 & $(-) 3.93$ & Excluded \\
\hline${ }^{*}$ GABRA1 & First Set & 0.605 & $(-) 6.12$ & Excluded \\
\hline GABRA2 & First Set & 0.575 & $(-) 20.25$ & Excluded \\
\hline GABRA6 & First Set & 0.646 & $(-) 10.69$ & Excluded \\
\hline *GABRD & First Set & 0.745 & $(-) 3.73$ & Excluded \\
\hline${ }^{*}$ GABRG2 & First Set & 0.773 & $(-) 22.33$ & Excluded \\
\hline${ }^{*}$ GABRG2 & Second Set & 0.455 & $(-) 4.39$ & Excluded \\
\hline${ }^{*} \mathrm{KCNA} 1$ & First Set & 0.716 & $(-) 6.71$ & Excluded \\
\hline KCNQ1 & First Set & 0.495 & $(-) 3.10$ & Excluded \\
\hline *KCNQ2 and CHRNA4 & Second Set & 0.578 & $(-) 1.50$ & Neither Linked Nor Unlinked \\
\hline *KCNQ2 and CHRNA4 & Third Set & 0.526 & $(-) 0.49$ & Neither Linked Nor Unlinked \\
\hline *KCNQ2 and CHRNA4 & Fourth Set & 0.676 & $(-) 11.37$ & Excluded \\
\hline *KCNQ3 & First Set & 0.711 & $(-) 2.01$ & Excluded \\
\hline KCNQ5 & First Set & 0.535 & $(-) 5.29$ & Excluded \\
\hline *LGI1 & First Set & 0.725 & $(-) 9.89$ & Excluded \\
\hline *ME2 & First Set & 0.625 & $(-) 7.89$ & Excluded \\
\hline
\end{tabular}


Table 1 Vizsla two-point linkage analysis results (Continued)

\begin{tabular}{|c|c|c|c|c|}
\hline *NHLRC1 & Second Set & 0.509 & $(-) 8.49$ & Excluded \\
\hline *SCN1A & First Set & 0.600 & $(-) 7.83$ & Excluded \\
\hline *SCN1B & First Set & 0.820 & $(-) 8.52$ & Excluded \\
\hline${ }^{*} \mathrm{SCN} 2 \mathrm{~A}$ & First Set & 0.833 & $(-) 13.95$ & Excluded \\
\hline SCN3A & First Set & 0.000 & $(-)$ & Monomorphic \\
\hline SCN3A & Second Set & 0.545 & $(-) 5.82$ & Excluded \\
\hline SCN3B & First Set & 0.751 & $(-) 21.76$ & Excluded \\
\hline SCN8A & First Set & 0.805 & $(-) 18.57$ & Excluded \\
\hline SCN11A & First Set & 0.255 & $(一)$ & Low Heterozygosity \\
\hline
\end{tabular}

Bolded genes marked with $\mathrm{a}$ * indicate those associated with human IE, and bolded genes marked with a \# indicate those associated with mouse models of epilepsy. The breed cohort was assembled and statistics were performed as described in Materials and Methods. "Designator" indicates each set of primers, first, second, etc., designed for that gene and used in this breed (See Additional files 1 and 2). $\mathrm{H}=$ heterozygosity of the marker.

gene, GABRA1, which is an inherited human epilepsy gene, initially resulted in marginally significant p-values for two different microsatellites: 0.074 (first set) and 0.033 (second set), however, a third microsatellite's alleles resulted in an insignificant $\mathrm{p}$-value of 0.890 . Additionally, when the Bonferroni correction was applied, these results were not significant; therefore, no further follow-up was initiated.

Results for the Greater Swiss Mountain Dogs are shown in Table 3. As with the ESS, Pearson chi-square p-values are reported for this association study. Again, the majority of the loci $(n=35)$ had highly insignificant p-values and are unlikely to be associated with IE in the GSMD; this included 17 of the 20 inherited human epilepsy-associated genes and both of the mouse model genes. This breed had the lowest overall average heterozygosity and results for eleven genes, three of which are human epilepsy-associated (GABRD, KCNA1, and ME2), could not be determined due to low heterozygosity. The first marker analyzed for $K C N D 2$ resulted in a p-value of 0.036 , prompting the examination of two additional markers. One of these was uninformative, but the other had a heterozygosity of 0.729 and a p-value of 0.155 , rendering this gene not significant and unlikely to be associated to IE in GSMD. Additionally, CHRNB2, a gene that is associated with human epilepsy, resulted in a p-value of 0.055 . While this p-value is potentially suggestive of association, when correcting for multiple testing with the Bonferroni correction, it does not approach significance.

Results for the Beagles are shown in Table 4. In this breed association study, highly insignificant $\mathrm{p}$-values for 43 candidate genes indicate that these genes are likely not associated with IE in Beagles. All twenty of the inherited human epilepsy-associated genes and both of the mouse model genes had insignificant p-values in this breed. Only three genes (CACNA1D, CACNG3, and $K C N Q 5)$ were inconclusive due to low heterozygosity in this breed. CHRNA1 gave a p-value of 0.05 on its initial marker, leading to the development of primers for additional microsatellites. Two of these, CHRNA1's third set and fourth set of primers, gave p-values of 0.072 and 0.056, respectively, but CHRNA1's second set of primers resulted in a highly insignificant $\mathrm{p}$-value of 0.600 , making it unlikely that this locus is associated with IE in this breed. Two other loci, both human epilepsy-associated genes, though insignificant, had lower p-values: KCNQ3 at 0.077 and LGI1 at 0.070. Again, applying correction for multiple testing indicates that these $\mathrm{p}$-values are not significant.

\section{Discussion}

This study was designed to use LD to exploit a possible founder effect suspected to be responsible for IE in these breeds by testing as many candidate genes as possible using microsatellite markers. Microsatellites have been shown to be a practical and useful tool in candidate gene studies [29]. Marker locations were selected within average blocks of canine LD so that one marker's result could, in theory, accurately represent the entire gene. The use of multiple breeds allowed for the possibility of assessing whether a discovered mutation was unique to one breed, suggesting the suspected founder effect specific to that breed, or was observed in several breeds, indicating a much older mutation. While no mutations were identified to draw definitive conclusions, it is not unreasonable to speculate that in dogs the underlying genetic basis of IE may vary by breed, as it is known that the rare human monogenic IEs are typically isolated by family [30-35]. These experiments incorporated both linkage analysis and association analysis, each having different strengths. While linkage analysis is the more powerful of these two statistical analysis methods for detecting Mendelian disease mutations, it requires samples from appropriate family structures. Conversely, association analysis does not require family-based samples, and it is generally considered more powerful than linkage analysis in detecting polygenic disease associations. Sequencing of the subset of human IE-associated candidate genes was not pursued for this study, as brain 
Table 2 English Springer Spaniel association chi-square results

\begin{tabular}{|c|c|c|c|c|}
\hline Gene Name & Designator & $\mathrm{H}$ & $\begin{array}{c}\text { Chi } \\
\text { Square } \\
\text { P-Value }\end{array}$ & Results \\
\hline${ }^{*} \mathrm{ARX}$ & First Set & 0.485 & 0.843 & Not Significant \\
\hline${ }^{*}$ CACNA1A & First Set & 0.312 & 0.308 & Not Significant \\
\hline CACNA1B & First Set & 0.062 & $(-)$ & $\begin{array}{c}\text { Low } \\
\text { Heterozygosity }\end{array}$ \\
\hline CACNA1D & First Set & 0.000 & $(-)$ & Monomorphic \\
\hline CACNA1E & First Set & 0.684 & 0.314 & Not Significant \\
\hline CACNA1F & First Set & 0.454 & 0.097 & Not Significant \\
\hline CACNA1G & First Set & 0.467 & 0.916 & Not Significant \\
\hline${ }^{*}$ CACNA1H & First Set & 0.734 & 0.644 & Not Significant \\
\hline CACNA1I & First Set & 0.546 & 0.135 & Not Significant \\
\hline \#CACNA2D2 & First Set & 0.369 & 0.898 & Not Significant \\
\hline CACNB1 & First Set & 0.734 & 0.744 & Not Significant \\
\hline CACNB2 & First Set & 0.619 & 0.305 & Not Significant \\
\hline CACNB3 & First Set & 0.362 & 0.113 & Not Significant \\
\hline${ }^{*}$ CACNB4 & First Set & 0.830 & 0.989 & Not Significant \\
\hline \#CACNG2 & First Set & 0.413 & 0.963 & Not Significant \\
\hline CACNG3 & First Set & 0.653 & 0.130 & Not Significant \\
\hline CACNG4 & First Set & 0.635 & 0.220 & Not Significant \\
\hline CACNG6 & First Set & 0.561 & 0.267 & Not Significant \\
\hline CHRNA1 & First Set & 0.538 & 0.249 & Not Significant \\
\hline${ }^{*}$ CHRNA2 & First Set & 0.233 & $(-)$ & $\begin{array}{c}\text { Low } \\
\text { Heterozygosity }\end{array}$ \\
\hline $\begin{array}{l}{ }^{*} \text { CHRNA4 } 4 \text { and } \\
\text { KCNQ2 }\end{array}$ & See KCNQ2 & & & \\
\hline CHRNA5 & First Set & 0.436 & 0.788 & Not Significant \\
\hline CHRNA7 & First Set & 0.692 & 0.622 & Not Significant \\
\hline CHRNA9 & First Set & 0.259 & $(-)$ & $\begin{array}{c}\text { Low } \\
\text { Heterozygosity }\end{array}$ \\
\hline CHRNB1 & First Set & 0.207 & $(-)$ & $\begin{array}{c}\text { Low } \\
\text { Heterozygosity }\end{array}$ \\
\hline${ }^{*}$ CHRNB2 & First Set & 0.173 & $(-)$ & $\begin{array}{c}\text { Low } \\
\text { Heterozygosity }\end{array}$ \\
\hline${ }^{*}$ CHRNB2 & Second Set & 0.198 & $(-)$ & $\begin{array}{c}\text { Low } \\
\text { Heterozygosity }\end{array}$ \\
\hline${ }^{*}$ CHRNB2 & Third Set & 0.321 & 0.982 & Not Significant \\
\hline${ }^{*} \mathrm{CLCN} 2$ & First Set & 0.492 & 0.719 & Not Significant \\
\hline DNM1 & First Set & 0.227 & $(-)$ & $\begin{array}{c}\text { Low } \\
\text { Heterozygosity }\end{array}$ \\
\hline DNM1 & Second Set & 0.528 & 0.886 & Not Significant \\
\hline DNM1 & Third Set & 0.700 & 0.598 & Not Significant \\
\hline DNM1 & Fourth Set & 0.714 & 0.875 & Not Significant \\
\hline DNM1 & Fifth Set & 0.618 & 0.853 & Not Significant \\
\hline *GABRA1 & First Set & 0.416 & 0.074 & Inconclusive \\
\hline *GABRA1 & Second Set & 0.490 & 0.033 & Inconclusive \\
\hline *GABRA1 & Third Set & 0.369 & 0.890 & Not Significant \\
\hline *GABRD & First Set & 0.257 & $(-)$ & $\begin{array}{c}\text { Low } \\
\text { Heterozygosity }\end{array}$ \\
\hline *GABRD & Second Set & 0.223 & $(-)$ & $\begin{array}{c}\text { Low } \\
\text { Heterozygosity }\end{array}$ \\
\hline *GABRD & Third Set & 0.295 & $(-)$ & $\begin{array}{c}\text { Low } \\
\text { Heterozygosity }\end{array}$ \\
\hline *GABRG2 & First Set & 0.663 & 0.443 & Not Significant \\
\hline
\end{tabular}

Table 2 English Springer Spaniel association chi-square results (Continued)

\begin{tabular}{|c|c|c|c|c|}
\hline${ }^{*} \mathrm{KCNA} 1$ & First Set & 0.587 & 0.965 & Not Significant \\
\hline KCND2 & First Set & 0.233 & $(-)$ & $\begin{array}{c}\text { Low } \\
\text { Heterozygosity }\end{array}$ \\
\hline $\begin{array}{l}\text { *KCNQ2 and } \\
\text { CHRNA4 }\end{array}$ & Second Set & 0.738 & 0.710 & Not Significant \\
\hline${ }^{*} \mathrm{KCNQ3}$ & First Set & 0.541 & 0.474 & Not Significant \\
\hline KCNQ5 & First Set & 0.206 & $(-)$ & $\begin{array}{c}\text { Low } \\
\text { Heterozygosity }\end{array}$ \\
\hline *LGI1 & First Set & 0.641 & 0.834 & Not Significant \\
\hline *ME2 & First Set & 0.492 & 0.619 & Not Significant \\
\hline${ }^{*}$ NHLRC1 & Second Set & 0.684 & 0.784 & Not Significant \\
\hline${ }^{*} \mathrm{SCN} 1 \mathrm{~A}$ & First Set & 0.199 & $(-)$ & $\begin{array}{c}\text { Low } \\
\text { Heterozygosity }\end{array}$ \\
\hline${ }^{*}$ SCN1A & Second Set & 0.082 & $(-)$ & $\begin{array}{c}\text { Low } \\
\text { Heterozygosity }\end{array}$ \\
\hline${ }^{*}$ SCN1A & Third Set & 0.211 & $(-)$ & $\begin{array}{c}\text { Low } \\
\text { Heterozygosity }\end{array}$ \\
\hline${ }^{*} \mathrm{SCN} 1 \mathrm{~A}$ & Fourth Set & 0.337 & 0.968 & Not Significant \\
\hline *SCN1B & First Set & 0.676 & 0.216 & Not Significant \\
\hline${ }^{*} \mathrm{SCN} 2 \mathrm{~A}$ & First Set & 0.766 & 0.670 & Not Significant \\
\hline SCN3A & First Set & 0.000 & $(-)$ & Monomorphic \\
\hline SCN3A & Second Set & 0.069 & $(-)$ & $\begin{array}{c}\text { Low } \\
\text { Heterozygosity }\end{array}$ \\
\hline SCN3B & First Set & 0.563 & 0.834 & Not Significant \\
\hline SCN8A & First Set & 0.070 & $(-)$ & $\begin{array}{c}\text { Low } \\
\text { Heterozygosity }\end{array}$ \\
\hline SCN8A & Second Set & 0.000 & $(-)$ & Monomorphic \\
\hline SCN11A & First Set & 0.637 & 0.396 & Not Significant \\
\hline
\end{tabular}

Table 3 Greater Swiss Mountain Dog association chisquare results

\begin{tabular}{lcccc}
\hline Gene Name & Designator & H & $\begin{array}{c}\text { Chi } \\
\text { Square } \\
\text { P-Value }\end{array}$ & Results \\
\hline *ARX & First Set & 0.396 & 0.606 & Not Significant \\
*CACNA1A & First Set & 0.000 & $(-)$ & Monomorphic \\
*CACNA1A & Second Set & 0.408 & 0.740 & Not Significant \\
CACNA1B & First Set & 0.370 & 0.905 & Not Significant \\
CACNA1D & First Set & 0.699 & 0.529 & Not Significant \\
CACNA1E & First Set & 0.675 & 0.953 & Not Significant \\
CACNA1F & First Set & 0.106 & $(-)$ & Low \\
CACNA1G & First Set & 0.000 & $(-)$ & Monomorphic \\
CACNA1G & Second Set & 0.023 & $(-)$ & Low \\
& & & & Heterozygosity \\
*CACNA1H & First Set & 0.000 & $(-)$ & Monomorphic \\
*CACNA1H & Second Set & 0.517 & 0.272 & Not Significant \\
CACNA11 & First Set & 0.483 & 0.449 & Not Significant \\
\#CACNA2D2 & First Set & 0.308 & 0.442 & Not Significant
\end{tabular}


Table 3 Greater Swiss Mountain Dog association chisquare results (Continued)

\begin{tabular}{|c|c|c|c|c|c|c|c|c|c|}
\hline CACNB1 & First Set & 0.261 & $(-)$ & $\begin{array}{l}\text { Low } \\
\text { Heterozygosity }\end{array}$ & $\begin{array}{l}{ }^{*} \mathrm{SCN} 1 \mathrm{~A} \\
{ }^{*} \mathrm{SCN} 1 \mathrm{~B}\end{array}$ & $\begin{array}{l}\text { First Set } \\
\text { First Set }\end{array}$ & $\begin{array}{l}0.749 \\
0.000\end{array}$ & $\begin{array}{l}0.706 \\
(-)\end{array}$ & $\begin{array}{l}\text { Not Significant } \\
\text { Monomorphic }\end{array}$ \\
\hline CACNB2 & First Set & 0.000 & $(-)$ & Monomorphic & *SCN1B & Second Set & 0.000 & $(-)$ & Monomorphic \\
\hline CACNB3 & First Set & 0.322 & 0.718 & Not Significant & ${ }^{*}$ SCN1B & Third Set & 0.026 & $(-)$ & Low \\
\hline *CACNB4 & First Set & 0.628 & 0.709 & Not Significant & & & & & Heterozygosity \\
\hline \#CACNG2 & First Set & 0.690 & 0.382 & Not Significant & *SCN1B & Fourth Set & 0.485 & 0.233 & Not Significant \\
\hline CACNG3 & First Set & 0.000 & $(-)$ & Monomorphic & ${ }^{*} \mathrm{SCN} 2 \mathrm{~A}$ & First Set & 0.686 & 0.767 & Not Significant \\
\hline CACNG3 & Second Set & 0.591 & 0.527 & Not Significant & SCN3A & First Set & 0.000 & $(-)$ & Monomorphic \\
\hline \multirow[t]{2}{*}{ CACNG4 } & First Set & 0.298 & $(-)$ & Low & SCN3A & Second Set & 0.437 & 0.378 & Not Significant \\
\hline & & & & Heterozygosity & SCN3B & First Set & 0.000 & $(-)$ & Monomorphic \\
\hline CACNG6 & First Set & 0.482 & 0.323 & Not Significant & SCN3B & Second Set & 0.000 & $(-)$ & Monomorphic \\
\hline CHRNA1 & First Set & 0.491 & 0.730 & Not Significant & SCN8A & First Set & 0.152 & $(-)$ & Low \\
\hline CHRNA1 & Second Set & 0.670 & 0.256 & Not Significant & & & & & Heterozygosity \\
\hline CHRNA1 & Third Set & 0.656 & 0.374 & Not Significant & SCN8A & Second Set & 0.576 & 0.996 & Not Significant \\
\hline CHRNA1 & Fourth Set & 0.452 & 0.582 & Not Significant & SCN11A & First Set & 0.597 & 0.967 & Not Significant \\
\hline${ }^{*}$ CHRNA2 & First Set & 0.461 & 0.644 & Not Significant & \multirow{4}{*}{\multicolumn{5}{|c|}{$\begin{array}{l}\text { Bolded genes marked with a * indicate those associated with human IE, and } \\
\text { bolded genes marked with a \# indicate those associated with mouse models } \\
\text { of epilepsy. The breed cohort was assembled and statistics were performed as } \\
\text { described in Materials and Methods. "Designator" indicates each set of } \\
\text { primers, first, second, etc., designed for that gene and used in this breed (See } \\
\text { Additional files } 1 \text { and 2). } \mathrm{H} \text { = heterozygosity of the marker. }\end{array}$}} \\
\hline $\begin{array}{l}\text { *CHRNA4 and } \\
\text { KCNQ2 }\end{array}$ & \multicolumn{4}{|l|}{ See KCNQ2 } & & & & & \\
\hline CHRNA5 & First Set & 0.389 & 0.483 & Not Significant & & & & & \\
\hline CHRNA7 & First Set & 0.810 & 0.924 & Not Significant & & & & & \\
\hline CHRNA9 & First Set & 0.615 & 0.875 & Not Significant & & & & & \\
\hline CHRNB1 & First Set & 0.000 & $(-)$ & Monomorphic & & & & & \\
\hline${ }^{*}$ CHRNB2 2 & First Set & 0.333 & 0.055 & Not Significant & \multicolumn{5}{|c|}{ Table 4 Beagle association chi-square results } \\
\hline \multirow{2}{*}{$\begin{array}{l}{ }^{*} \mathrm{CLCN} 2 \\
\text { DNM1 }\end{array}$} & First Set & 0.519 & 0.600 & Not Significant & \multirow{2}{*}{ Gene Name } & \multirow{2}{*}{ Designator } & \multirow[t]{2}{*}{$\mathrm{H}$} & \multirow{2}{*}{$\begin{array}{l}\text { Chi } \\
\text { Square } \\
\text { P-Value }\end{array}$} & \multirow[t]{2}{*}{ Results } \\
\hline & First Set & 0.139 & $(-)$ & $\begin{array}{l}\text { Low } \\
\text { Heterozygosity }\end{array}$ & & & & & \\
\hline DNM1 & Second Set & 0.364 & 0.799 & Not Significant & ${ }^{*} \mathrm{ARX}$ & First Set & 0.508 & 0.643 & Not Significant \\
\hline DNM1 & Third Set & 0.500 & 0.834 & Not Significant & ${ }^{*}$ CACNA1A & First Set & 0.636 & 0.813 & Not Significant \\
\hline DNM1 & Fourth Set & 0.000 & $(-)$ & Monomorphic & CACNA1B & First Set & 0.836 & 0.765 & Not Significant \\
\hline DNM1 & Fifth Set & 0.484 & 0.464 & Not Significant & CACNA1D & First Set & 0.123 & $(-)$ & Low \\
\hline *GABRA1 & First Set & 0.549 & 0.354 & Not Significant & & & & & Heterozygosity \\
\hline *GABRD & First Set & 0.088 & $(-)$ & Low & CACNA1E & First Set & 0.697 & 0.254 & Not Significant \\
\hline & & & & erozygosity & CACNA1F & First Set & 0.671 & 0.578 & Not Significant \\
\hline *GABRD & Second Set & 0.088 & $(-)$ & $\begin{array}{l}\text { Low } \\
\text { Heterozygosity }\end{array}$ & CACNA1G & First Set & 0.640 & 0.092 & Not Significant \\
\hline${ }^{*}$ GABRD & Third Set & 0.081 & $(-)$ & & ${ }^{*}$ CACNA1H & Second Set & 0.344 & 0.873 & Not Significant \\
\hline & & & & Heterozygosity & CACNA1I & First Set & 0.570 & 0.365 & Not Significant \\
\hline *GABRG2 & First Set & 0.219 & $(-)$ & & \#CACNA2D2 & First Set & 0.438 & 0.917 & Not Significant \\
\hline & & & & Heterozygosity & CACNB1 & First Set & 0.318 & 0.661 & Not Significant \\
\hline *GABRG2 & Second Set & 0.458 & 0.929 & Not Significant & CACNB2 & First Set & 0.612 & 0.577 & Not Significant \\
\hline *KCNA1 & First Set & 0.171 & $(-)$ & Low & CACNB3 & First Set & 0.577 & 0.148 & Not Significant \\
\hline & & & & Heterozygosity & *CACNB4 & First Set & 0.864 & 0.753 & Not Significant \\
\hline KCND2 & First Set & 0.480 & 0.036 & Inconclusive & \#CACNG2 & First Set & 0.720 & 0.502 & Not Significant \\
\hline KCND2 & Second Set & 0.729 & 0.155 & Not Significant & CACNG3 & First Set & 0.135 & $(-)$ & Low \\
\hline KCND2 & Third Set & 0.124 & $(-)$ & Low & & & & & Heterozygosity \\
\hline${ }^{*} \mathrm{KCNQ} 2$ and & First Set & 0.441 & 0.677 & Not Sianificant & CACNG4 & First Set & 0.633 & 0.816 & Not Significant \\
\hline CHRNA4 & & & & Not Signiricant & CACNG6 & First Set & 0.505 & 0.413 & Not Significant \\
\hline *KCNQ3 & First Set & 0.382 & 0.904 & Not Significant & CHRNA1 & First Set & 0.686 & 0.050 & Not Significant \\
\hline KCNQ5 & First Set & 0.100 & $(-)$ & & CHRNA1 & Second Set & 0.506 & 0.600 & Not Significant \\
\hline & & & & Heterozygosity & CHRNA1 & Third Set & 0.661 & 0.072 & Not Significant \\
\hline *LGI1 & First Set & 0.440 & 0.244 & Not Significant & CHRNA1 & Fourth Set & 0.790 & 0.056 & Not Significant \\
\hline *ME2 & First Set & 0.219 & $(-)$ & Low & ${ }^{*}$ CHRNA2 & First Set & 0.664 & 0.590 & Not Significant \\
\hline & & & & Heterozygosity & *CHRNA & See KCNQ2 & & & \\
\hline *NHLRC1 & Second Set & 0.405 & 0.240 & Not Significant & KCNQ2 & & & & \\
\hline
\end{tabular}

Table 3 Greater Swiss Mountain Dog association chi-

square results (Continued)

Bolded genes marked with $a$ * indicate those associated with human IE, and primers, first, second, etc., designed for that gene and used in this breed (See

(1)


Table 4 Beagle association chi-square results (Continued)

\begin{tabular}{|c|c|c|c|c|}
\hline CHRNA7 & First Set & 0.741 & 0.513 & Not Significant \\
\hline CHRNA9 & First Set & 0.665 & 0.694 & Not Significant \\
\hline CHRNB1 & First Set & 0.491 & 0.555 & Not Significant \\
\hline${ }^{*}$ CHRNB2 & First Set & 0.583 & 0.885 & Not Significant \\
\hline${ }^{*} \mathrm{CLCN} 2$ & First Set & 0.762 & 0.577 & Not Significant \\
\hline DNM1 & First Set & 0.278 & $(-)$ & $\begin{array}{c}\text { Low } \\
\text { Heterozygosity }\end{array}$ \\
\hline DNM1 & Second Set & 0.386 & 0.693 & Not Significant \\
\hline DNM1 & Third Set & 0.588 & 0.314 & Not Significant \\
\hline DNM1 & Fourth Set & 0.565 & 0.953 & Not Significant \\
\hline DNM1 & Fifth Set & 0.563 & 0.430 & Not Significant \\
\hline *GABRA1 & First Set & 0.523 & 0.345 & Not Significant \\
\hline GABRA2 & First Set & 0.502 & 0.265 & Not Significant \\
\hline GABRA6 & First Set & 0.575 & 0.266 & Not Significant \\
\hline *GABRD & First Set & 0.684 & 0.554 & Not Significant \\
\hline *GABRG2 & First Set & 0.748 & 0.292 & Not Significant \\
\hline *GABRG2 & Second Set & 0.447 & 0.849 & Not Significant \\
\hline *KCNA1 & First Set & 0.758 & 0.356 & Not Significant \\
\hline KCND2 & First Set & 0.530 & 0.919 & Not Significant \\
\hline KCND2 & Second Set & 0.706 & 0.698 & Not Significant \\
\hline KCND2 & Third Set & 0.728 & 0.455 & Not Significant \\
\hline $\begin{array}{l}\text { *KCNQ2 and } \\
\text { CHRNA4 }\end{array}$ & Second Set & 0.805 & 0.627 & Not Significant \\
\hline *KCNQ3 & First Set & 0.655 & 0.077 & Not Significant \\
\hline KCNQ5 & First Set & 0.106 & $(-)$ & $\begin{array}{c}\text { Low } \\
\text { Heterozygosity }\end{array}$ \\
\hline *LGI1 & First Set & 0.234 & $(-)$ & $\begin{array}{c}\text { Low } \\
\text { Heterozygosity }\end{array}$ \\
\hline *LGI1 & Second Set & 0.708 & 0.070 & Not Significant \\
\hline *ME2 & First Set & 0.585 & 0.282 & Not Significant \\
\hline${ }^{*}$ NHLRC1 & First Set & 0.605 & 0.739 & Not Significant \\
\hline *SCN1A & First Set & 0.118 & $(-)$ & $\begin{array}{c}\text { Low } \\
\text { Heterozygosity }\end{array}$ \\
\hline${ }^{*} \mathrm{SCN} 1 \mathrm{~A}$ & Third Set & 0.328 & 0.551 & Not Significant \\
\hline *SCN1B & First Set & 0.679 & 0.425 & Not Significant \\
\hline *SCN1B & Second Set & 0.649 & 0.885 & Not Significant \\
\hline *SCN2A & First Set & 0.680 & 0.763 & Not Significant \\
\hline SCN3B & First Set & 0.750 & 0.127 & Not Significant \\
\hline SCN8A & First Set & 0.730 & 0.110 & Not Significant \\
\hline SCN11A & First Set & 0.689 & 0.572 & Not Significant \\
\hline
\end{tabular}

Bolded genes marked with $a$ * indicate those associated with human $\mathrm{IE}$, and bolded genes marked with a \# indicate those associated with mouse models of epilepsy. The breed cohort was assembled and statistics were performed as described in Materials and Methods. "Designator" indicates each set of primers, first, second, etc., designed for that gene and used in this breed (See Additional files 1 and 2). $\mathrm{H}=$ heterozygosity of the marker.

tissues for obtaining cDNAs for most cost efficient sequencing were not available.

Overall marker informativeness was highly influenced by sample population. The Vizslas and Beagles had excellent average heterozygosities $(0.569$ and 0.574 , respectively), while the ESS and GSMD were much lower ( 0.425 and 0.341 , respectively). This may be due, in part, to the sample cohorts: the ESS and GSMD were set up as discordant full- or half-sibling pairs, whereas the Vizslas were constructed as large pedigrees, and the Beagles were case/control matched pairs that did not share common ancestors to at least the grandparent level. Reduced marker heterozygosity across breeds could also be influenced by a higher degree of inbreeding within the ESS and GSMD sample cohorts in this study, and the lower average heterozygosities of these two breeds may suggest a founder effect that could eventually help uncover associations. However, marker informativeness is not entirely dependent on sample population, as evidenced by $C A C N A 1 A$, which was polymorphic enough to be confidently insignificant after analyzing one or two microsatellites in ESS, GSMD, and Beagles, but remained inconclusive through three markers in the Vizslas and ultimately required a fourth microsatellite to achieve a suitable heterozygosity in this breed. The accepted marker heterozygosity of 0.3 is perhaps low, especially when analyzing only a single microsatellite for each candidate gene, however, for many of the insignificant microsatellite results in all four breeds the heterozygosity was $>0.5$.

Ultimately, 16 of the 20 human epilepsy-associated candidate genes (ARX, CACNA1A, CACNA1H, CACNB4, CHRNA4, CHRNB2, CLCN2, GABRA1, GABRG2, KCNQ2, KCNQ3, LGI1, NHLRC1, SCN1A, SCN1B, and $S C N 2 A)$ and both of the mouse model genes (CACNA2D2 and $C A C N G 2$ ) were either excluded (with linkage) or demonstrated insignificant association to IE in all four of the breed cohorts. Another two of them (KCNA1 and $M E 2)$ were insignificant in three out of four breed cohorts, remaining inconclusive only in the GSMD. One gene, $G A B R D$, was inconclusive in two breeds (ESS and GSMD), and CHRNA2 was inconclusive in ESS and was not tested in Vizslas. Insignificant association to IE was demonstrated in most of the additional candidate genes tested. For every breed, however, there were a handful of markers that were inconclusive, due to low heterozygosity.

The few markers with potentially interesting results (uncorrected $\mathrm{p}$-values of $<0.05$ ) that were not excluded by follow-up markers, including $C A C N B 1$ in the Vizsla, CHRNB2 in the GSMD, and KCNQ3 and LGI1 in the Beagle, were of minimal interest when correcting for multiple testing. With the Bonferroni correction, a p-value of 0.05 considered as statistically significant would be lowered to 0.0025 for twenty tests, and further lowered to 0.00125 for forty tests; p-values of this magnitude were not obtained for any marker, and all breeds were tested on more than forty genes. The potential of false positives due to population stratification must also be considered for these few genes with suggestive results in the association studies, despite attempts to control for the degree of relatedness. Haplotype association analysis was not possible with this data since there were not 
enough closely-spaced markers to generate haplotypes. Conclusive confirmation or exclusion of these loci can be performed in the future with newer technologies such as whole-genome SNP arrays, which can generate vast amounts of data in less time.

It is possible that the populations tested were underpowered to detect association if it existed. Utilizing a siblingpair case-control design could decrease power because the control dog may also carry the risk allele. However, this situation is difficult to avoid in highly inbred dog populations, and the sibling-pair case-control design should aid in avoiding population stratification, which can create false positive results. Although sample size requirements for canine association studies have not been precisely defined, based on average linkage disequilibrium estimates in dog breeds, a starting point of approximately 25 cases and 25 controls appears to be adequate to find statistical significance for a completely penetrant recessive trait [36]. The number increases to approximately 50 cases and 50 controls when a trait is dominant, and for more complex traits as many as 100 dogs in each group may be necessary. Therefore, it is possible that there were not adequate numbers of dogs in the association studies, particularly if IE is not monogenic. A meta-analysis might improve power by pooling data from the three association study breeds (ESS, GSMD, and Beagle). However, this proved to be impractical because 1) many microsatellites had varying informativeness in each breed, so that pooling data would only work for a single microsatellite that was informative for a gene in all three breeds and 2) microsatellites by their very nature are highly polymorphic and there was often little overlap in alleles between breeds. Lastly, if IE has a different genetic basis in each of these breeds, pooling data across breeds seems unlikely to yield interesting results.

In the linkage analysis of Vizsla pedigrees, it is possible to conclude that these candidate gene loci are truly excluded, due to convincingly negative LOD scores at zero centiMorgans. However, if the inheritance and age dependent penetrance assumptions for the Vizsla model are incorrect, then the present linkage study is flawed. These input assumptions were based on previous study of IE in this breed [23] and on the best information available. Conversely, in the association studies of the other three breeds, it is not possible to specifically state that the loci are "excluded", as there is some degree of doubt that one microsatellite marker is adequate to provide convincing evidence for exclusion of a locus with this type of study. The addition of more markers would help to verify these insignificant results and truly exclude a locus. That work is beyond the scope of this study and is better addressed with whole-genome SNP analysis. The latest commercial canine SNP array, with over 170,000 SNPs, became available well after these studies were initiated. Further, the excluded and insignificant genes reported in the present study may only reflect the specific breeds examined, or even lines within these breeds, and results should not necessarily be extrapolated to other breeds.

It is possible that canine IE, like the majority of human IE, is a genetically complex disease in most breeds and that multiple loci contribute to susceptibility in any given breed. The present study would very likely have detected a major contributing locus, and it is unlikely that a truly causative locus is being excluded as a false negative. In humans, the vast majority of IE remains genetically unexplained and is considered to be polygenic [37-39]. A recent study by Oberbauer et al. [40] utilized microsatellites in a genome-wide linkage scan for epilepsy loci in the Belgian shepherd dog and concluded that the disease was highly polygenic, reporting a tentative six QTLs. These results further support the conclusion that canine IE is a more complex disease than originally hypothesized. Whole-genome association analyses with SNP arrays are likely the platform of choice for further studies of IE, as they can query tens of thousands of markers simultaneously across the genome and are better able to identify multiple susceptibility loci. Additionally, copy number variants (CNVs) have been increasingly shown to be involved in neurologic disorders such as autism [41,42] and schizophrenia $[43,44]$, as well as epilepsy [45]. CNV studies of canine epilepsy may reveal this as a similar mechanism for disease in both species.

Ultimately, canine IE found to be significantly associated with DNA markers or with a mutation in a specific gene would allow genetic tests to be developed to assist dog breeders with decreasing the incidence of this disease. This would be most effective for monogenic, highly penetrant forms of IE, but if the disease proves to be genetically complex, with multiple genes contributing and less than $100 \%$ penetrance, then such a test could still possibly be used to provide a relative risk for IE development within a breed. In any event, discovery of IE associated gene loci in dogs may not only improve the understanding of canine health, but could advance the study of neurobiology and human health as well.

\section{Conclusions}

The present study did not identify any obvious association or linkage between IE and the microsatellite markers tested for the various candidate genes in any of the four dog breeds. It is possible that IE may in fact be a polygenic disorder even in highly inbred dog breeds, which underscores the challenging nature of investigating inherited epilepsy. Eventually identifying the genetic causes for IE in dogs would have a significant impact on canine health, as well as providing a useful model for IE in humans. 


\section{Methods \\ Case Definition}

Since seizure activity can result from numerous discernable abnormalities, and only dogs very likely to be affected with IE were to be included in the study, the case definition was designed to eliminate, as much as possible, all other likely causes of seizures. Therefore, in order to qualify for "case" status for IE, each affected dog must have had two or more seizures with clinical normalcy between episodes, a veterinarian-administered normal physical examination and neurological examination, as well as normal bloodwork, including a complete blood count and blood chemistry profile, and, when possible, a bile acids assay. Since IE has been shown to be statistically much more probable when the dog is aged 1 to 5 years at the time of its first seizure [46], only dogs whose first seizure occurred during this time frame were included. If seizures started prior to 1 year of age or after 5 years of age, extra criteria were then required to enroll a dog, including a normal MRI or CT, and/or normal CSF analysis. EEG was not used as a case criterion due to its limited availability in clinical veterinary practice. To be classified as unaffected, the dog must have reached at least five years of age and be completely free of known seizure activity. In the situation where a case was identified at a young age, and its full- or half-sibling was used as a control, the dogs were followed for years until the unaffected dog had reached at least five years of age.

Dogs in these studies were privately owned and voluntarily enrolled by their owners with informed consent. Cases were solicited through breed publications, presentations at local and national breed club meetings, and word of mouth. For each enrolled dog, a general health survey, a signed owner consent form, and up to ten mLs of whole blood in an EDTA tube were required. Blood samples were obtained via venipuncture either at the dog's regular veterinarian or at specially-organized blood-draws at breed shows across the USA. This study was approved by the University of Minnesota IACUC.

Owners of dogs affected with seizures were asked to fill out an additional questionnaire which asked for information about the dog's seizure characteristics, preictal and postictal signs, previous medical history, and any anti-epileptic drug therapy; these were submitted by phone, fax, postal mail or e-mail. Any additional followup questions were conducted via telephone interview. Finally, medical records for each affected dog were obtained from the dog's regular veterinarian and any specialists (if applicable); these were inspected for bloodwork results and the veterinarian's physical and neurological examination findings. Dogs were assigned case, control, or unknown status after careful evaluation of this information. "Unknown" status was reserved for those dogs displaying suspicious seizure or seizure-like activity, but couldn't be classified as a case for various reasons; for example, if the dog had only one recorded seizure or had suffered head trauma prior to onset of the first seizure.

\section{DNA Isolation}

DNA was isolated from whole blood following standard protocols, utilizing the Puregene DNA Isolation Kit from Qiagen Inc. (Valencia, CA).

\section{Breed Cohorts}

Cohorts of affected and unaffected dogs for genotyping were assembled for each of the four test breeds - the Vizsla, the GSMD, the ESS, and the Beagle. Ultimately, the available samples determined what type of genetic analysis was conducted for each breed.

\section{Vizslas}

For Vizslas, the depth of the available DNA samples from multiple siblings, parents, and many grandparents allowed the development of multi-generation pedigrees (Figure 1 and Figure 2) suitable for genetic linkage analysis. 96 dogs were included: 31 affected dogs, 60 unaffected dogs, and 5 dogs with unknown phenotype status. These dogs were separated into two large family pedigrees shown in Figures 1 and 2, and were subsequently broken into ten smaller families to decrease inbreeding loops for linkage analysis and calculation of LOD scores.

\section{Greater Swiss Mountain Dogs}

For the GSMD, discordant full-sibling or half-sibling pairs of affected $(n=24)$ and unaffected $(n=24)$ dogs were assembled and used in association studies. Siblings were used to help avoid spurious association from population stratification. In a few instances, two different pairs of siblings were related to each other, such as sharing one parent.

\section{English Springer Spaniels}

Similar to the GSMD, the ESS were assembled into discordant full- or half-sibling pairs of affected $(\mathrm{n}=24)$ and unaffected $(\mathrm{n}=24)$ dogs and were used in association studies. As in the GSMD, occasionally the pairs of siblings were also related to one another; this was impossible to avoid due to the inbred nature of the breed.

\section{Beagles}

Association studies were also conducted for the Beagles. Due to lack of available sibling pairs, pedigree analyses were used to ensure that only dogs with no common 

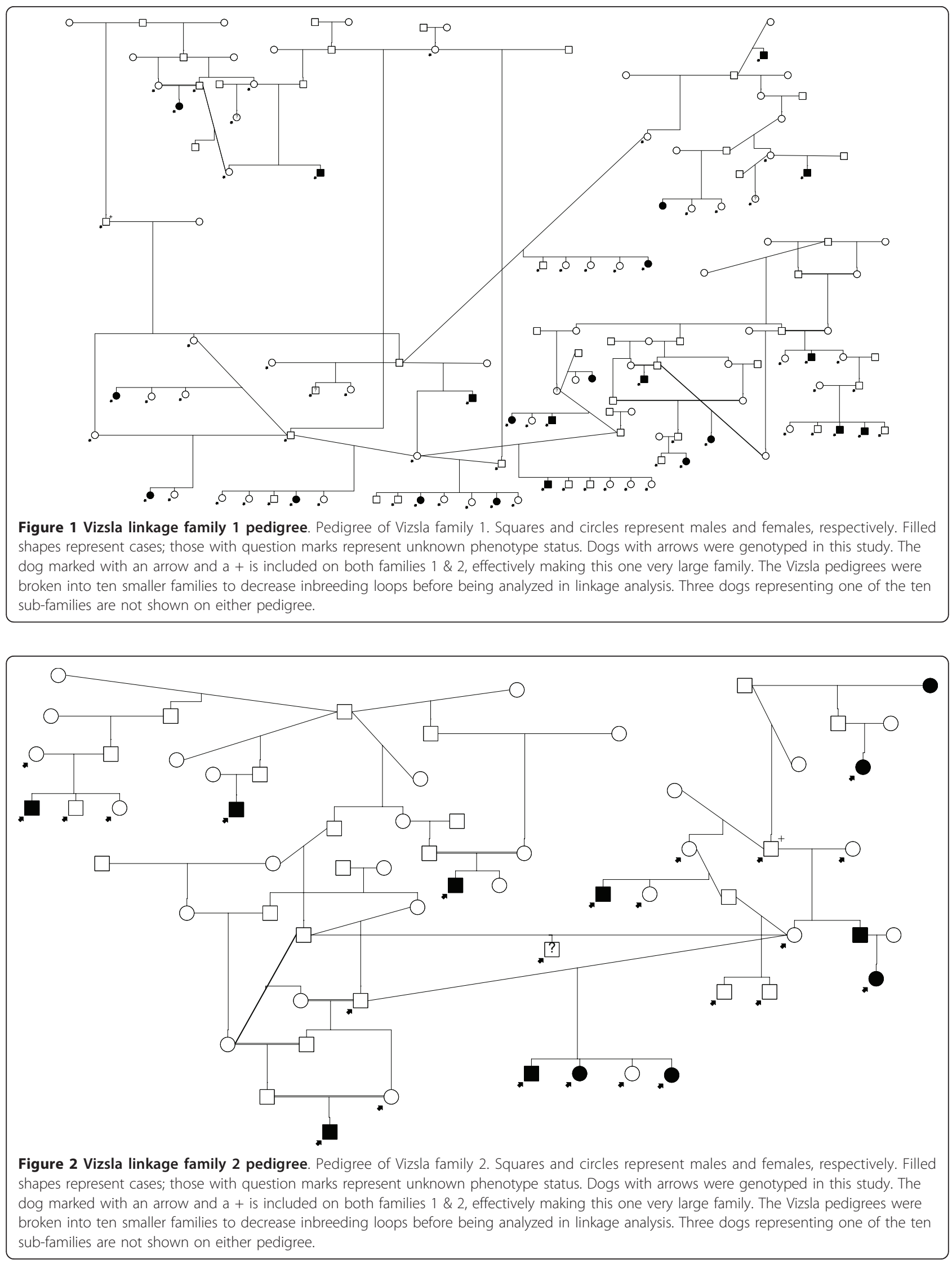
grandparents were utilized. This strategy has previously shown to be adequate for canine genetic mutation detection [47]. Twenty-four affected dogs and 24 unaffected dogs were selected for this dataset.

\section{Candidate Gene Selection}

A non-exhaustive list of candidate genes known to be involved with inherited human epilepsy was assembled, including potassium channels KCNA1, KCNQ2, and KCNQ3; calcium channels CACNA1A, CACNA1H, and CACNB4; sodium channels SCN1A, SCN2A, and SCN1B; GABA receptors GABRA1, GABRD, and GABRG2; acetylcholine receptors CHRNA2, CHRNA4, and CHRNB2; and chloride channel CLCN2; as well as novel (ie, nonion channel and non-neurotransmitter receptor) genes malic enzyme 2 (ME2), leucine-rich glioma inactivated 1 (LGI1), NHL repeat containing 1 (NHLRC1), and aristaless related homeobox $(A R X)$, for a total of 20 human epilepsy-associated genes [30,48-54]. Two candidate genes associated with mouse epilepsy models were also included: CACNA2D2, (the ducky mouse) [55] and CACNG2 (the stargazer mouse) [56].

In addition to this core set of genes, further genes were selected from the same gene families based on their presence in neural tissue, including: calcium channels CACNA1B, CACNA1D, CACNA1E, CACNA1F, CACNA1G, CACNA1I, CACNB1, CACNB2, CACNB3, CACNG3, CACNG4, and CACNG6; sodium channels $S C N 3 A, S C N 3 B, S C N 8 A$, and $S C N 11 A$; GABA receptors GABRA2, and GABRA6; acetylcholine receptors CHRNA1, CHRNA3, CHRNA5, CHRNA9, CHRNA10, CHRNB1, and CHRND as well as CHRNA7, which has had suggested linkage with human IE [57]; and potassium channels KCND2, KCNQ1, and KCNQ5. Finally, the dynamin-1 gene (DNM1) was also examined. This gene was recently found to be mutated in the canine disease termed exercise induced collapse [58] and a mutation in dynamin-1 has recently been characterized in the fitful mouse model, wherein heterozygotes for the mutation experience recurrent seizures and homozygotes for the mutation often have lethal seizures and other neurosensory deficits [59]. Therefore, a total of 52 candidate genes were chosen for this study.

\section{Primer design, PCR, and Genotyping}

Human mRNA sequence was obtained for each gene from the National Center for Biotechnology Information [60]. This sequence was BLATed on the University of California - Santa Cruz Genome Browser website [61] against the May 2005 dog genome assembly to identify the resultant genomic position of each gene in the dog. Microsatellites were selected either within the gene or closely flanking the gene utilizing the Variation and Repeat - Microsatellite feature of this browser. After capturing the microsatellite's flanking sequence, primer pairs to amplify each microsatellite were designed using Primer3 [62]. An additional 5' tail sequence was added to each right (reverse) primer [63] to generate fluorescent labels in PCR. All primer sequences are shown in Additional file 1, and Additional file 2 lists the Mb positions of each gene and microsatellite. Every attempt was made to select microsatellites less than $1 \mathrm{Mb}$ distant from the gene, in order to remain within estimated blocks of canine linkage disequilibrium (LD) [36] for each gene. LD is the non-random association of alleles at two loci and, within dog breeds, can extend over distances of several megabases [36]. However, for many of the candidate genes, several microsatellites (and therefore several sets of primers) needed to be tested due to the microsatellite's lack of informativeness (monomorphism or low heterozygosity), and eventually the only useable microsatellite was located further away. Additional file 2 indicates which microsatellites $(\mathrm{n}=3)$ were $>1 \mathrm{Mb}$ distant. CHRNA4 and KCNQ2 were close enough to one another in the canine genome that one marker was used for both genes.

Each microsatellite was PCR amplified in $15-\mu \mathrm{L}$ reactions including $12.5 \mathrm{ng}$ canine DNA, 10X PCR Buffer, $2.5 \mathrm{pmol}$ of the forward primer and $0.75 \mathrm{pmol}$ of the reverse-tailed primer, $0.78 \mathrm{pmol}$ of a fluorescent dyelabeled primer complementary to the tail sequence, 20 $\mu \mathrm{M}$ dNTP mix, and 0.5 units of Taq DNA polymerase (Qiagen Hot StarTaq, Qiagen Inc, Valencia, CA), with $\mathrm{dH}_{2} \mathrm{O}$ to final volume. PCR cycling conditions were $95^{\circ}$ $\mathrm{C}$ for 20 minutes, then 40 cycles of the following: $94^{\circ} \mathrm{C}$ for 30 seconds, $58^{\circ} \mathrm{C}$ for 30 seconds, and $72^{\circ} \mathrm{C}$ for 30 to 60 seconds (depending on product size), and a final extension of $72^{\circ} \mathrm{C}$ for 15 minutes. PCR products were prepared for capillary gel electrophoresis according to manufacturer's recommendations and size separated using the Beckman CEQ 8000 automated DNA-fragment analyzer with fluorescence detection (Beckman Coulter Inc, Fullerton CA). Genotype data were analyzed with instrument software, and alleles were manually verified.

\section{Statistical Analysis}

Marker heterozygosity was calculated using the following formula: $H=1-\Sigma p_{i}{ }^{2}$, where $p_{i}$ is the population frequency of the $i$ th allele, and $H$ is the probability that a random individual is heterozygous for any two alleles at a gene locus with allele frequencies, $p_{i}$ [64]. A cut-off of 0.3 was selected for heterozygosity; markers with heterozygosity falling below this cut-off were not considered informative enough to confidently claim a non-significant result for a gene, since less than one third of all individuals would be expected to be heterozygous at that locus [64]. 
In order to determine the power of the ten Vizsla family pedigrees to detect linkage, simulated linkage was performed with the FASTSLINK program [65-67]. Multiple parameters were used, including combinations of 3 and 4 equally frequent alleles, 0 and $5 \%$ heterogeneity (proportion of unlinked families) and recombination frequencies of 0.01 and 0.001 . Recombination frequencies were kept very small since the microsatellites tested were within or very near to the candidate genes. Two hundred simulations were performed for each set of parameters, assuming autosomal recessive inheritance.

Parametric two-point linkage analysis was utilized for Vizsla marker data, with significant evidence for linkage a LOD (logarithm of odds) score of 3.2 or greater [68]. A LOD score of less than -2.0 was considered to exclude a locus. Input assumptions were an autosomal recessive mode of inheritance with $95 \%$ penetrance for the disease genotype, and a disease allele frequency of 0.20 ; these assumptions were based on previously published data concerning IE in this breed [23]. Two-point LOD scores were calculated using the FASTLINK version of MLINK [69-72].

For the GSMD, ESS, and Beagles, the allele frequencies were subjected to a nonparametric chi-square test for independence (association analysis) [73]. Alleles with frequencies of less than $10 \%$ (minor alleles) were removed before conducting the chi-square analysis. A Pearson chi-square p-value of 0.05 was first used as the level of significance necessary to detect significantly different allele frequencies between affected and unaffected dogs. Subsequently, a Bonferroni corrected p-value of 0.001 , correcting for $\sim 52$ tests, was considered evidence for strong association. Power analyses were not specifically conducted for these three breeds, as sample size requirements for canine association studies have not been precisely defined.

\section{Additional material}

\section{Additional file 1: Primer sequences for candidate gene}

microsatellites. Sequence information and product sizes are presented for primers designed for microsatellites examined for each candidate gene. Bolded genes marked with $a *$ indicate those associated with human epilepsy, and bolded genes marked with a \# indicate those associated with mouse models of epilepsy. Two of the DNM1 marker primer pairs, DNM1 Third Set and DNM1 Fifth Set, are taken from the University of California-Davis Canine Genetic Linkage Map [74], and are named therein 0945 and 0946, respectively. CFA = canis familiaris chromosome.

Additional file 2: Candidate gene locations and primer locations. All pertinent canine chromosomal location information is presented for each gene and all microsatellites examined for that gene. Bolded genes marked with a * indicate those associated with human epilepsy, and bolded genes marked with a \# indicate those associated with mouse models of epilepsy. Two of the DNM1 marker primer pairs, DNM1 Third Set and DNM1 Fifth Set, are taken from the University of California-Davis Canine Genetic Linkage Map [74], and are named therein 0945 and 0946, respectively. $\wedge$ Bolded entries in this column indicate microsatellites located outside the gene itself and further than $1 \mathrm{Mb}$ away $(n=3)$. Mb positions given for microsatellites are the first nucleotide of each microsatellite. Mb positions were taken from Ensembl [75] and the UCSC Genome Browser [61]. CFA = canis familiaris chromosome.

\section{Acknowledgements}

This work was supported by the National Institutes of Health [RR18719] and the American Kennel Club Canine Health Foundation [598]. The contents of this publication are solely those of the authors and do not necessarily reflect the views of the Canine Health Foundation. The authors gratefully acknowledge Dr. Gary Johnson and Liz Hansen of the University of Missouri for supplying the GSMD DNA samples. This work is dedicated to the memory of Monica C. Roberts, who was a major contributor to the initiation of this project.

\section{Author details}

${ }^{1}$ Department of Veterinary Clinical Sciences, College of Veterinary Medicine, University of Minnesota, 1352 Boyd Avenue, Saint Paul, Minnesota, 55108 , USA. ${ }^{2}$ Department of Veterinary and Biomedical Sciences, College of Veterinary Medicine, University of Minnesota, 295 ASNM, 1988 Fitch Avenue, St. Paul, Minnesota, 55108, USA.

\section{Authors' contributions}

KJE participated in conceiving the study, carried out the methods including choosing microsatellites, designing primers, genotyping, statistical analyses, and drafted the manuscript. EEP conceived the study and study design, and coordinated sample accession. KMM participated in carrying out methods including constructing pedigrees and genotyping, as well as sample accession. JRM conceived the study and study design and contributed significant manuscript review. All authors read and approved the final manuscript.

Received: 17 December 2010 Accepted: 25 April 2011

Published: 25 April 2011

\section{References}

1. Sander JW: The epidemiology of epilepsy revisited. Curr Opin Neurol 2003, 16(2):165-70.

2. Jallon P, Latour P: Epidemiology of idiopathic generalized epilepsies. Epilepsia 2005, 46(Suppl 9):10-4.

3. Noebels $\mathrm{J}$ : Exploring new gene discoveries in idiopathic generalized epilepsy. Epilepsia 2003, 44(Suppl 2):16-21.

4. Scheffer IE, Berkovic SF: The genetics of human epilepsy. Trends Pharmacol Sci 2003, 24(8):428-33.

5. Gourfinkel-An I, Baulac S, Nabbout R, Ruberg M, Baulac M, Brice A, LeGuern E: Monogenic idiopathic epilepsies. Lancet Neurol 2004, 3(4):209-218.

6. Steinlein OK: Genes and mutations in human idiopathic epilepsy. Brain Dev 2004, 26(4):213-8.

7. Lu Y, Wang X: Genes associated with idiopathic epilepsies: a current overview. Neurol Res 2009, 31(2):135-43.

8. Berendt $M$, Gredal H, Alving J: Characteristics and phenomenology of epileptic partial seizures in dogs: similarities with human seizure semiology. Epilepsy Res 2004, 61:167-73.

9. Leppik IE, Patterson E, Hardy B, Cloyd JC: Canine status epilepticus: Proof of principle studies. Epilepsia 2009, 50(Suppl 12):14-5.

10. Chandler K: Canine epilepsy: what can we learn from human seizure disorders? Vet J 2006, 172(2):207-17.

11. Knowles K: Idiopathic epilepsy. Clin Tech Small Anim Pract 1998, 13(3):144-51.

12. Bielfelt SW, Redman HC, McClellan RO: Sire- and sex-related differences in rates of epileptiform seizures in a purebred beagle dog colony. Am J Vet Res 1971, 32(12):2039-48

13. Falco MJ, Barker J, Wallace ME: The genetics of epilepsy in the British Alsatian. J Small Anim Pract 1974, 15(11):685-92.

14. Hall SJ, Wallace ME: Canine epilepsy: a genetic counselling programme for keeshonds. Vet Rec 1996, 138(15):358-60.

15. Jaggy A, Faissler D, Gaillard C, Srenk P, Graber H: Genetic aspects of idiopathic epilepsy in Labrador retrievers. J Small Anim Pract 1998, 39(6):275-80. 
16. Srenk P, Jaggy A, Gaillard C, Busato A, Horin P: Genetic basis of idiopathic epilepsy in the golden retriever. Tierarztl Prax 1994, 22(6):574-8.

17. Srenk $P$, Jaggy $A$ : Interictal electroencephalographic findings in a family of golden retrievers with idiopathic epilepsy. J Small Anim Pract 1996, 37(7):317-21

18. Kathmann I, Jaggy A, Busato A, Bartschi M, Gaillard C: Clinical and genetic investigations of idiopathic epilepsy in the Bernese mountain dog. $J$ Small Anim Pract 1999, 40(7):319-25

19. Famula TR, Oberbauer AM, Brown KN: Heritability of epileptic seizures in the Belgian tervueren. J Small Anim Pract 1997, 38(8):349-52.

20. Famula TR, Oberbauer AM: Segregation analysis of epilepsy in the Belgian tervueren dog. Vet Rec 2000, 147(8):218-21.

21. Nielen $A L$, Janss $L L$, Knol BW: Heritability estimations for diseases, coat color, body weight, and height in a birth cohort of Boxers. Am J Vet Res 2001, 62(8):1198-206

22. Morita T, Shimada A, Takeuchi T, Hikasa Y, Sawada M, Ohiwa S, Takahashi M, Kubo N, Shibahara T, Miyata H, Ohama E: Cliniconeuropathologic findings of familial frontal lobe epilepsy in Shetland sheepdogs. Can J Vet Res 2002, 66(1):35-41.

23. Patterson EE, Mickelson JR, Da Y, Roberts MC, McVey AS, O'Brien DP Johnson GS, Armstrong PJ: Clinical characteristics and inheritance of idiopathic epilepsy in Vizslas. J Vet Intern Med 2003, 17(3):319-25.

24. Patterson EE, Armstrong PJ, O'Brien DP, Roberts MC, Johnson GS, Mickelson JR: Clinical description and mode of inheritance of idiopathic epilepsy in English springer spaniels. J Am Vet Med Assoc 2005, 226(1):54-8.

25. Casal ML, Munuve RM, Janis MA, Werner P, Henthorn PS: Epilepsy in Irish Wolfhounds. J Vet Intern Med 2006, 20(1):131-5.

26. Licht BG, Lin S, Luo Y, Hyson LL, Licht MH, Harper KM, Sullivan SA Fernandez SA, Johnston EV: Clinical characteristics and mode of inheritance of familial focal seizures in Standard Poodles. $J$ Am Vet med Assoc 2007, 231(10):1520-8.

27. Lohi H, Young EJ, Fitzmaurice SN, Rusbridge C, Chan EM, Vervoort M, Turnbull J, Zhao XC, lanzano L, Paterson AD, Sutter NB, Ostrander EA, Andre C, Shelton GD, Ackerley CA, Scherer SW, Minassian BA: Expanded repeat in canine epilepsy. Science 2005, 307(5706):81.

28. Ostrander EA, Kruglyak L: Unleashing the canine genome. Genome Res 2000, 10(9):1271-4

29. Mellersh C: Microsatellite-based candidate gene linkage analysis studies. Methods Mol Biol 2008, 439:75-86

30. Aridon P, Marini C, Di Resta C, Brilli E, De Fusco M, Politi F, Parrini E, Manfredia I, Pisano T, Pruna D, Curia G, Cianchetti C, Pasqualetti M, Becchetti A, Guerrini R, Casari G: Increased sensitivity of the neuronal nicotinic receptor alpha 2 subunit causes familial epilepsy with nocturnal wandering and ictal fear. Am J Hum Genet 2006, 79(2):342-50.

31. Phillips HA, Scheffer IE, Berkovic SF, Hollway GE, Sutherland GR, Mulley JC: Localization of a gene for autosomal dominant nocturnal frontal lobe epilepsy to chromosome 20q 13.2. Nat Genet 1995, 10(1):117-8.

32. Charlier C, Singh NA, Ryan SG, Lewis TB, Reus BE, Leach RJ, Leppert M: A pore mutation in a novel KQT-like potassium channel gene in an idiopathic epilepsy family. Nat Genet 1998, 18(1):53-5.

33. Wallace RH, Wang DW, Singh $R$, Scheffer IE, George AL Jr, Phillips HA, Saar K, Reis A, Johnson EW, Sutherland GR, Berkovic SF, Mulley JC: Febrile seizures and generalized epilepsy associated with a mutation in the $\mathrm{Na}$ +-channel beta1 subunit gene SCN1B. Nat Genet 1998, 19(4):366-70.

34. Escayg A, MacDonald BT, Meisler MH, Baulac S, Huberfeld G, An-Gourfinkel I, Brice A, LeGuern E, Moulard B, Chaigne D, Buresi C, Malafosse A: Mutations of SCN1A, encoding a neuronal sodium channel, in two families with GEFS+2. Nat Genet 2000, 24(4):343-5.

35. Weber YG, Lerche H: Genetic mechanisms in idiopathic epilepsies. Dev Med Child Neurol 2008, 50(9):648-54

36. Lindblad-Toh K, Wade CM, Mikkelsen TS, Karlsson EK, Jaffe DB, Kamal M, Clamp M, Chang JL, Kulbokas EJ, Zody MC, Mauceli E, Xie X, Breen M, Wayne RK, Ostrander EA, Ponting CP, Galibert F, Smith DR, DeJong PJ, Kirkness E, Alvarez P, Biagi T, Brockman W, Butler J, Chin CW, Cook A, Cuff J, Daly MJ, DeCaprio D, Gnerre S, Grabherr M, Kellis M, Kleber M, Bardeleben C, Goodstadt L, Heger A, Hitte C, Kim L, Koepfli KP, Parker HG, Pollinger JP, Searle SM, Sutter NB, Thomas R, Webber C, Baldwin J, Abebe A, Abouelleil A, Aftuck L, Ait-Zahra M, Aldredge T, Allen N, An P, Anderson S, Antoine C, Arachchi $H$, Aslam A, Ayotte L, Bachantsang P, Barry A, Bayul T, Benamara M, Berlin A, Bessette D, Blitshteyn B, Bloom T,
Blye J, Boguslavskiy L, Bonnet C, Boukhgalter B, Brown A, Cahill P, Calixte N, Camarata J, Cheshatsang Y, Chu J, Citroen M, Collymore A, Cooke P, Dawoe T, Daza R, Decktor K, DeGray S, Dhargay N, Dooley K, Dooley K, Dorje P, Dorjee K, Dorris L, Duffey N, Dupes A, Egbiremolen O, Elong R, Falk J, Farina A, Faro S, Ferguson D, Ferreira P, Fisher S, FitzGerald M, Foley K, Foley C, Franke A, Friedrich D, Gage D, Garber M, Gearin G, Giannoukos G, Goode T, Goyette A, Graham J, Grandbois E, Gyaltsen K, Hafez N, Hagopian D, Hagos B, Hall J, Healy C, Hegarty R, Honan T, Horn A, Houde N, Hughes L, Hunnicutt L, Husby M, Jester B, Jones C, Kamat A, Kanga B, Kells C, Khazanovich D, Kieu AC, Kisner P, Kumar M, Lance K, Landers T, Lara M, Lee W, Leger JP, Lennon N, Leuper L, LeVine S, Liu J, Liu X, Lokyitsang Y, Lokyitsang T, Lui A, Macdonald J, Major J, Marabella R, Maru K, Matthews C, McDonough S, Mehta T, Meldrim J, Melnikov A, Meneus L, Mihalev A, Mihova T, Miller K, Mittelman R, Mlenga V, Mulrain L, Munson G, Navidi A, Naylor J, Nguyen T, Nguyen N, Nguyen C, Nguyen T, Nicol R, Norbu N, Norbu C, Novod N, Nyima T, Olandt P, O'Neill B, O'Neill K, Osman S, Oyono L, Patti C, Perrin D, Phunkhang P, Pierre F, Priest M, Rachupka A, Raghuraman S, Rameau R, Ray V, Raymond C, Rege F, Rise C, Rogers J, Rogov P, Sahalie J, Settipalli S, Sharpe T, Shea T, Sheehan M, Sherpa N, Shi J, Shih D, Sloan J, Smith C, Sparrow T, Stalker J, StangeThomann N, Stavropoulos S, Stone C, Stone S, Sykes S, Tchuinga P, Tenzing P, Tesfaye S, Thoulutsang D, Thoulutsang Y, Topham K, Topping I, Tsamla T, Vassiliev H, Venkataraman $V$, Vo A, Wangchuk T, Wangdi T, Weiand M, Wilkinson J, Wilson A, Yadav S, Yang S, Yang X, Young G, Yu Q, Zainoun J, Zembek L, Zimmer A, Lander ES: Genome sequence, comparative analysis and haplotype structure of the domestic dog Nature 2005, 438(7069):803-19.

37. Steinlein OK: New insights into the molecular and genetic mechanisms underlying idiopathic epilepsies. Clin Genet 1998, 54(3):169-75.

38. Heron SE, Scheffer IE, Berkovic SF, Dibbens LM, Mulley JC: Channelopathies in idiopathic epilepsy. Neurotherapeutics 2007, 4(2):295-304.

39. Sanchez-Carpintero Abad R, Sanmarti Vilaplana FX, Serratosa JM: Genetic Causes of Epilepsy. Neurologist 2007, 13(6 Suppl 1):S47-S51.

40. Oberbauer AM, Belanger JM, Grossman DI, Regan KR, Famula TR: Genomewide linkage scan for loci associated with epilepsy in Belgian shepherd dogs. BMC Genet 2010, 11:35.

41. Kumar RA, KaraMohamed S, Sudi J, Conrad DF, Brune C, Badner JA, Gilliam TC, Nowak NJ, Cook EH Jr, Dobyns WB, Christian SL: Recurrent 16p11.2 microdeletions in autism. Hum Mol Genet 2008, 17(4):628-38

42. Weiss LA, Shen Y, Korn JM, Arking DE, Miller DT, Fossdal R, Saemundsen E, Stefansson H, Ferreira MA, Green T, Platt OS, Ruderfer DM, Walsh CA, Altshuler D, Chakravarti A, Tanzi RE, Stefansson K, Santangelo SL, Gusella JF, Sklar P, Wu BL, Daly MJ, Autisim Consortium: Association between microdeletion and microduplication at 16p11.2 and autism. N Engl J Med 2008, 358(7):667-75.

43. Walsh T, McClellan JM, McCarthy SE, Addington AM, Pierce SB, Cooper GM, Nord AS, Kusenda M, Malhotra D, Bhandari A, Stray SM, Rippey CF, Roccanova P, Makarov V, Lakshmi B, Findling RL, Sikich L, Stromberg T, Merriman B, Gogtay N, Butler P, Eckstrand K, Noory L, Gochman P, Long R, Chen Z, Davis S, Baker C, Eichler EE, Meltzer PS, Nelson SF, Singleton AB, Lee MK, Rapoport JL, King MC, Sebat J: Rare structural variants disrupt multiple genes in neurodevelopmental pathways in schizophrenia. Science 2008, 320(5875):539-43.

44. Xu B, Roos JL, Levy S, van Rensburg EJ, Gogos JA, Karayiorgou M: Strong association of de novo copy number mutations with sproadic schizophrenia. Nat Genet 2008, 40(7):880-5.

45. Mefford HC, Muhle H, Ostertag P, von Spiczak S, Buysse K, Baker C, Franke A, Malafosse A, Genton P, Thomas P, Gurnett CA, Schreiber S, Bassuk AG, Guipponi M, Stephani U, Helbig I, Eichler EE: Genome-wide copy number variation in epilepsy: novel susceptibility loci in idiopathic generalized and focal epilepsies. PLoS Genet 2010, 6(5):e1000962.

46. Podell M, Fenner WR, Powers JD: Seizure classification in dogs from a nonreferral-based population. J Am Vet Med Assoc 1995, 206(11):1721-8.

47. Cadieu E, Neff MW, Quignon P, Walsh K, Chase K, Parker HG, Vonholdt BM, Rhue A, Boyko A, Byers A, Wong A, Mosher DS, Elkahloun AG, Spady TC, Andre C, Lark KG, Cargill M, Bustamante CD, Wayne RK, Ostrander EA: Coat variation in the domestic dog is governed by variants in three genes. Science 2009, 326(5949):150-3.

48. Chan EM, Young EJ, lanzano L, Munteanu I, Zhao X, Christopoulos CC, Avanzini G, Elia M, Ackerley CA, Jovic NJ, Bohlega S, Andermann E, Rouleau GA, Delgado-Escueta AV, Minassian BA, Scherer SW: Mutations in 
NHLRC1 cause progressive myoclonus epilepsy. Nat Genet 2003, 35(2):125-7.

49. Gardiner M: Genetics of idiopathic generalized epilepsies. Epilepsia 2005, 46(Suppl 9):15-20.

50. Turnbull J, Lohi H, Kearney JA, Rouleau GA, Delgado-Escueta AV, Meisler MH, Cossette P, Minassian BA: Sacred disease secrets revealed: the genetics of human epilepsy. Hum Mol Genet 2005, 14(17):2491-500.

51. Gomez-Abad C, Gomez-Garre P, Gutierrez-Delicado E, Saygi S, Michelucci R, Tassinari CA, Rodriguez de Cordoba S, Serratosa JM: Lafora disease due to EPM2B mutations: a clinical and genetic study. Neurology 2005, 64(6):982-6.

52. Gurnett CA, Hedera P: New ideas in epilepsy genetics: novel epilepsy genes, copy number alterations, and gene regulation. Arch Neurol 2007, 64(3):324-8.

53. Kato M, Saitoh S, Kamei A, Shiraishi H, Ueda Y, Akasaka M, Tohyama J, Akasaka N, Hayasaka K: A longer polyalanine expansion mutation in the ARX gene causes early infantile epileptic encephalopathy with suppression-burst pattern (Ohtahara syndrome). Am J Hum Genet 2007. 81(2):361-6.

54. Wallerstein $R$, Sugalski $R$, Cohn $L$, Jawetz $R$, Friez M: Expansion of the ARX spectrum. Clin Neurol Neurosurg 2008, 110(6):631-4.

55. Barclay J, Balaguero N, Mione M, Ackerman SL, Letts VA, Brodbeck J, Canti C, Meir A, Page KM, Kusumi K, Perez-Reyes E, Lander ES, Frankel WN Gardiner RM, Dolphin AC, Rees M: Ducky mouse phenotype of epilepsy and ataxia is associated with mutations in the Cacna2 $\mathrm{d} 2$ gene and decreased calcium channel current in cerebellar Purkinje cells. J Neurosci 2001, 21(16):6095-104.

56. Frankel WN: Detecting genes in new and old mouse models for epilepsy: a prospectus through the magnifying glass. Epilepsy Res 1999, 36:97-110.

57. Elmslie FV, Rees M, Williamson MP, Kerr M, Kjeldsen MJ, Pang KA, Sundqvist A, Friis ML, Chadwick D, Richens A, Covanis A, Santos M, Arzimanoglou A, Panayiotopoulos CP, Curtis D, Whitehouse WP, Gardiner RM: Genetic mapping of a major susceptibility locus for juvenile myoclonic epilepsy on chromosome 15q. Hum Mol Genet 1997, 6(8):1329-34.

58. Patterson EE, Minor KM, Tchernatynskaia AV, Taylor SM, Shelton GD, Ekenstedt KJ, Mickelson JR: A canine DNM1 mutation is highly associated with the syndrome of exercise-induced collapse. Nat Genet 2008, 40(10):1235-9.

59. Boumil RM, Letts VA, Roberts MC, Lenz C, Mahaffey CL, Zhang ZW, Moser T, Frankel WN: A missense mutation in a highly conserved alternate exon of dynamin-1 causes epilepsy in fitful mice. PLoS Genet 2010, 6(8): e1001046.

60. National Center for Biotechnology Information. [http://www.ncbinlm.nih. gov/].

61. UC Santa Cruz Genome Browser Home. [http://genome.ucscedu/].

62. Primer3. [http://frodo.wi.mit.edu/].

63. Oetting WS, Armstrong CM, Ronan SM, Young TL, Sellers TA, King RA: Multiplexed short tandem repeat polymorphisms of the Weber $8 \mathrm{~A}$ set of markers using tailed primers and infrared fluorescence detection. Electrophoresis 1998, 19(18):3079-83.

64. Ott J: Analysis of Human Genetic Linkage. 3 edition. Baltimore: Johns Hopkins University Press; 1999.

65. Ott J: Computer-simulation methods in human linkage analysis. Proc Natl Acad Sci USA 1989, 86(11):4175-8.

66. Weeks DE, Ott J, Lathrop GM: SLINK: a general simulation program for linkage analysis [abstract]. Am J Hum Genet 1990, 47(A204).

67. Cottingham RW Jr, Idury RM, Schaffer AA: Faster sequential genetic linkage computations. Am J Hum Genet 1993, 53(1):252-63.

68. Gordon D, Corwin MB, Mellersh CS, Ostrander EA, Ott J: Establishing appropriate genome-wide significance levels for canine linkage analysis. $J$ Hered 2003, 94(1):1-7.

69. Lathrop GM, Lalouel JM: Easy calculations of lod scores and genetic risks on small computers. Am J Hum Genet 1984, 36(2):460-5.

70. Lathrop GM, Lalouel JM, Julier C, Ott J: Strategies for multilocus linkage analysis in humans. Proc Natl Acad Sci USA 1984, 81(11):3443-6.

71. Lathrop GM, Lalouel JM, White RL: Construction of human linkage maps: likelihood calculations for multilocus linkage analysis. Genet Epidemiol 1986, 3(1):39-52

72. Schaffer AA: Faster linkage analysis computations for pedigrees with loops or unused alleles. Hum Hered 1996, 46(4):226-35.
73. Moore DS, McCabe GP: Introduction to the Practice of Statistics. 5 edition. New York: WH Freeman and Company; 2006.

74. UC Davis Canine Genetic Linkage Map. [http://www.vgl.ucdavis.edu/ dogmap].

75. Dog (Canis familiaris) Ensembl Website. [http://www.ensembl.org/ canis_familiaris/].

doi:10.1186/1471-2156-12-38

Cite this article as: Ekenstedt et al:: Candidate genes for idiopathic epilepsy in four dog breeds. BMC Genetics 2011 12:38.

\section{Submit your next manuscript to BioMed Central and take full advantage of:}

- Convenient online submission

- Thorough peer review

- No space constraints or color figure charges

- Immediate publication on acceptance

- Inclusion in PubMed, CAS, Scopus and Google Scholar

- Research which is freely available for redistribution

Submit your manuscript at www.biomedcentral.com/submit
Biomed Central 